\title{
Molecular characteristics of oxazolidinone resistance in enterococci from a multicenter study in China
}

\author{
Hongbin Chen ${ }^{\dagger}$, Xiaojuan Wang ${ }^{\dagger}$, Yuyao Yin, Shuguang Li, Yawei Zhang, Qi Wang and Hui Wang ${ }^{*}$ (D)
}

\begin{abstract}
Background: Linezolid-resistant enterococci pose great challenges in clinical practice. The aim of this study is to study the mechanisms underlying the resistance and genetic environment of antimicrobial resistance gene of linezolid-resistant enterococci.

Results: The linezolid MICs of 16 enterococci were $4 \mathrm{mg} / \mathrm{L}$ to $16 \mathrm{mg} / \mathrm{L}$. Four strains belonged to multi-drug resistant (MDR) bacteria. The sequence types (STs) of 13 enterococci strains performed WGS were diverse: 3 ST476, 1 ST86, ST116, ST480, ST59, ST416, ST21, ST67, ST16, ST585 and ST18. None of them carried multi-drug resistance gene cfr. Only one strain had the G2658 T mutation of target $23 S$ rRNA gene. Thirteen (13/16, 81.3\%) strains harbored the novel oxazolidinone resistance gene optrA. WGS analysis showed that the optrA gene was flanked by sequence IS1216E insertion in 13 strains, and optrA was adjacent to transposons Tn558 in two strains and Tn554 in one strain. The optrA gene was identified to be co-localized with fexA, the resistance genes mediated florfenicol resistance in 13 strains, and ermA1, the resistance genes mediated erythromycin resistance in 9 strains, indicating that linezolidresistant strains may be selected due to non-oxazolidinone antibiotics (i.e. macrolides and florfenicol) usage.
\end{abstract}

Conclusion: Our findings demonstrate the high diversity of optrA-carrying genetic platforms. The mobile genetic elements (MGEs) may play an important role in the dissemination of optrA into the enterococci isolates of human origin. The genetic evidence of transferable feature and co-selection of optrA should be gave more attention in clinical practice.

Keywords: optrA, Linezolid resistance, Oxazolidinone, Enterococci, Genetic environment

\section{Background}

Linezolid, which belongs to oxazolidinone, is the clinically last resort to treat vancomycin-resistant enterococci (VRE), methicillin-resistant Staphylococcus aureus (MRSA), and other multi-drug Gram-positive bacteria [1]. Linezolid exerts antibacterial effects by inhibiting the binding of mRNA to the ribosome, thereby affecting the synthesis of the protein [1]. It is generally considered that linezolid is a completely synthetic antibiotic, and theoretically, there should be no natural resistance phenomenon. Unfortunately, clinically resistant strains have emerged shortly after use of linezolid in clinical practice [2, 3]. The occurrence of linezolid-resistant strains show an increasing

\footnotetext{
* Correspondence: whuibj@163.com

${ }^{+}$Hongbin Chen and Xiaojuan Wang contributed equally to this work. Department of Clinical Laboratory, Peking University People's Hospital, Beijing 100044, People's Republic of China
}

trend, especially in animal husbandry [4], which should attract sufficient attention.

The resistance to linezolid by gram-positive bacteria can be achieved by target-modified 23S rRNA mutations [5], acquiring exogenous chloramphenicol-florfenicol resistance (cfr) [6], optrA [7] or poxtA [8]. Targets 23S rRNA, L3, L4 and L22 mutations usually affect ribosome function and easily reverse in the absence of selective pressure. Therefore, chemical modifications (such as methylation) of rRNA are the more common resistance mechanisms of linezolid. The $c f r$ gene encodes a methyltransferase that modifies the 23S rRNA at position A2503, which confers resistance to phenicols, lincosamide, oxazolidinones, pleuromutilin, and streptogramin A $\left(\mathrm{PhLOPS}_{\mathrm{A}}\right.$ phenotype) [9]. The cfr gene has been identified in a variety of genera, including Staphylococcus [10], Bacillus [11], Enterococcus [12], Macrococcus [13],

(c) The Author(s). 2019 Open Access This article is distributed under the terms of the Creative Commons Attribution 4.0 International License (http://creativecommons.org/licenses/by/4.0/), which permits unrestricted use, distribution, and 
Jeotgalicoccus [13], Streptococcus [14], Proteus [15] and Escherichia [16]. The cfr gene widely disseminates among oxazolidinone-resistant isolates from human [17] and animal [18] origin, which represents a serious threat to public health. Recently, two $c f r$ variants, $c f r(\mathrm{~B})$ and $c f r(\mathrm{C})$, have been found in Enterococcus faecium [19], Clostridium difficile [20] and Campylobacter [21]. The cfr gene was often found on a number of different plasmids [7, 15, 22], and integrated into transposons, leading to dissemination of this gene among the same or between different species of bacteria.

The transferable gene, optrA, has been identified, which confers cross-resistance to phenicols and oxazolidinones, including tedizolid [23]. This gene was identified in enterococci and staphylococci from clinical [24], healthy human and animal isolates $[25,26]$. The resistance gene optrA can be located either on plasmid or chromosome [26]. Recently, one florfenicol-resistant Staphylococcus sciuri isolate, which carried both optrA and $c f r$, was identified in pig [27]. In this study, we investigated the oxazolidinones resistance genes among linezolid-resistant isolates in Chinese hospitals and utilized whole-genome sequencing (WGS), and further analyzed the genetic environment surrounding the resistance genes.

\section{Materials and methods}

\section{Bacterial strains}

A total of 15 non-duplicable linezolid-resistant enterococci strains and one linezolid intermediate-resistant enterococci strain (13 E. faecalis and 3 E. faecium) $(1.5 \%, 16 / 1067)$ were collected from specimens of 16 patients from 9 hospitals between 2009 and 2013 in 6 provinces of China, including 5 samples from Beijing, 4 samples from Guangdong, 3 samples from Zhejiang, 2 samples from Fujian, 1 sample from Jiangsu and 1 sample from Hubei (Table 1.). Among the 16 strains, 6 were recovered from patients with urinary tract infection, 5 from patients with bacteremia, 4 from patients with wound infection and 1 from patients with biliary tract infection. Among the 16 strains, 7 strains (1203_10W003, 1202_13E004, 1202 21W014, 19113, 19677, 19506 and SZ21494) were isolated in our previous study [28], and the 9 remaining strains were isolated in this study. Bacteria were first identified at the species level using the VITEK system (bioMerieux, Crapome, France), followed by a molecular method based on the $16 \mathrm{~S}$ rRNA gene, and then by sequencing analysis.

\section{Antimicrobial susceptibility testing}

The minimal inhibitory concentrations (MICs) of 8 antimicrobial agents were determined by the agar dilution method, and tigecycline and daptomycin by broth microdilution. The antimicrobial agents tested included linezolid (Sigma Chemical Co., St. Louis, MO, USA), vancomycin (Sigma), teicoplanin (Sigma), levofloxacin
(Sigma), erythromycin (Sigma), tigecycline (Pfizer, NY, USA), daptomycin (Cubist Pharmaceuticals, MA, USA), penicillin (Sigma), ampicillin (Sigma) and gentamycin (Sigma). E. faecalis ATCC 29212 was used for quality control in antimicrobial susceptibility testing. The results of susceptibility testing were interpreted according to CLSI guideline M100-S27. Isolates resistant to three or more antibiotics of different families were considered to be multi-drug resistant (MDR).

\section{Molecular detection of resistance genes and mutations}

The resistance genes $c f r$ and optrA were determined by PCR as described previously. The mutation of domain V of the 23S rRNA gene was determined by PCR combined with sequencing as described previously [29]. Nucleotide sequences were compared with the linezolidsusceptible E. faecalis and E. faecium from Peking University People's Hospital during the same period. The mutation was identified by the $E$. coli numbering.

\section{Whole-genome sequencing (WGS)}

Total genomic DNA of 13 enterococci strains carrying optrA gene was extracted by the standard phenol/ chloroform method. The whole-genome sequencing was performed using Illumina technology. The sequences with read length of 150 bases were assembled into contigs using SPAdes (v.3.9.0) [30]. Plasmid content associated with optrA was analyzed using the contigs obtained by plasmidSPAdes. The assembled contigs were annotated by the Prokka v1.12 [31]. Insertion sequences (IS) were identified using ISFinder [32]. Multilocus sequence types (MLST) were assigned using the silico tool hosted by Center for Genomic Epidemiology (CGE) (www.genomicepidemiology.org). The resistance genes were identified by ResFinder 3.0 [33]. Maximum likelihood phylogenetic analysis of the core genome was performed using RAxML (Linux version v7.2.8) [34]. The sequences of the optrA-containing regions of 13 enterococci strains have been deposited at GenBank under the following accession numbers MH225413 (1202_13E004), MH225414 (1202_21W014), MH225415 (1203_10W003), MH225416 (1207_26W003), MH225417 (19506), MH225418 (19677), MH225419 (29462), MH225420 (SZ21494), MH225421 (TZ2), MH225422 (WHXH), MH225423 (XM2013_42321), MH225424 (XM2013_71028) and MH225425 (ZJ11066).

\section{Results}

Susceptibility profiles of linezolid-resistant enterococci isolates

The susceptible breakpoint of enterococci to linezolid is defined as less than or equal to $2 \mathrm{mg} / \mathrm{L}$, and the resistant breakpoint is defined as greater than or equal to $8 \mathrm{mg} / \mathrm{L}$. The linezolid MICs of 16 enterococci were 4 $\mathrm{mg} / \mathrm{L}$ to $16 \mathrm{mg} / \mathrm{L}$, respectively. There were no significant 


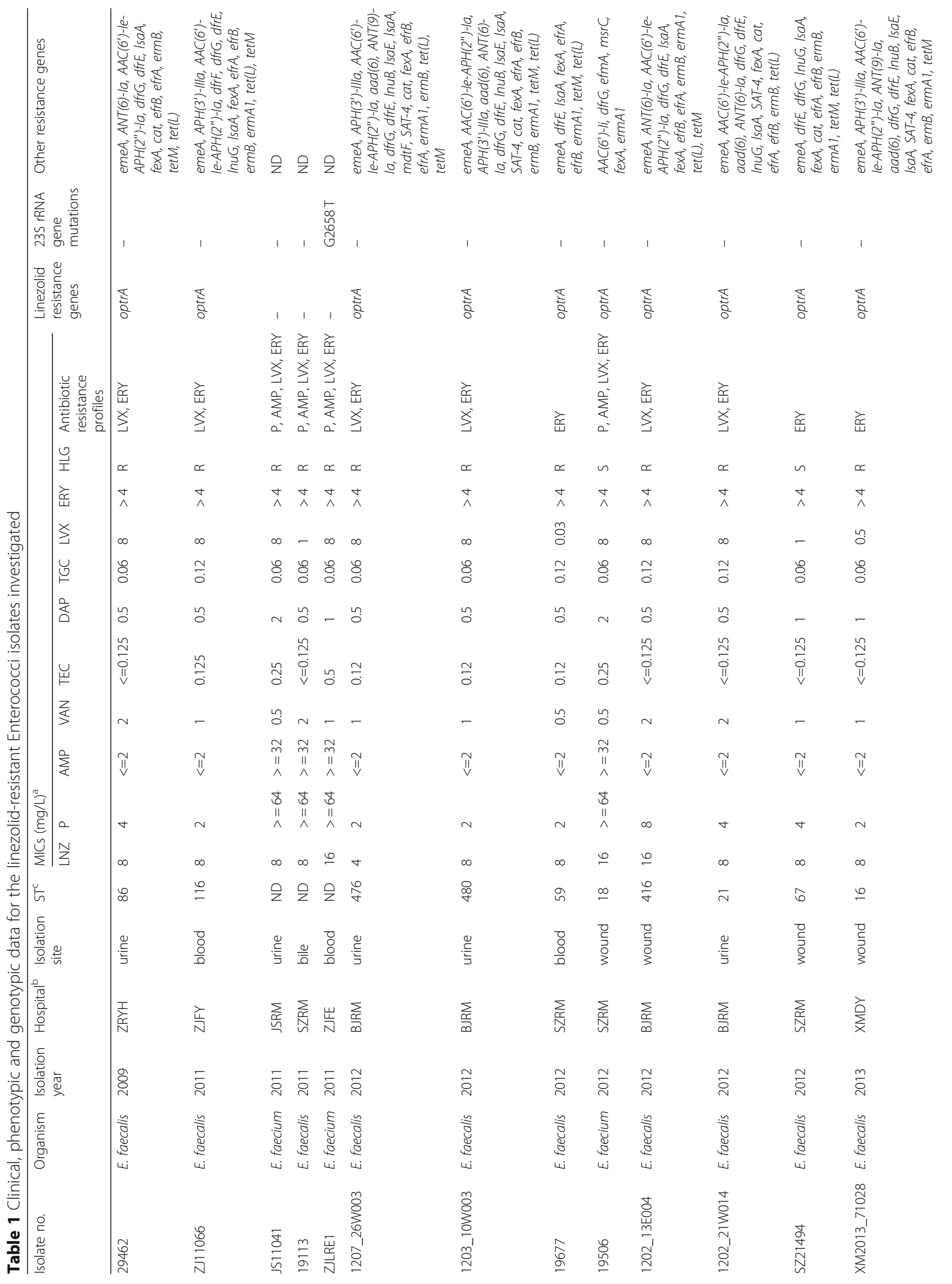




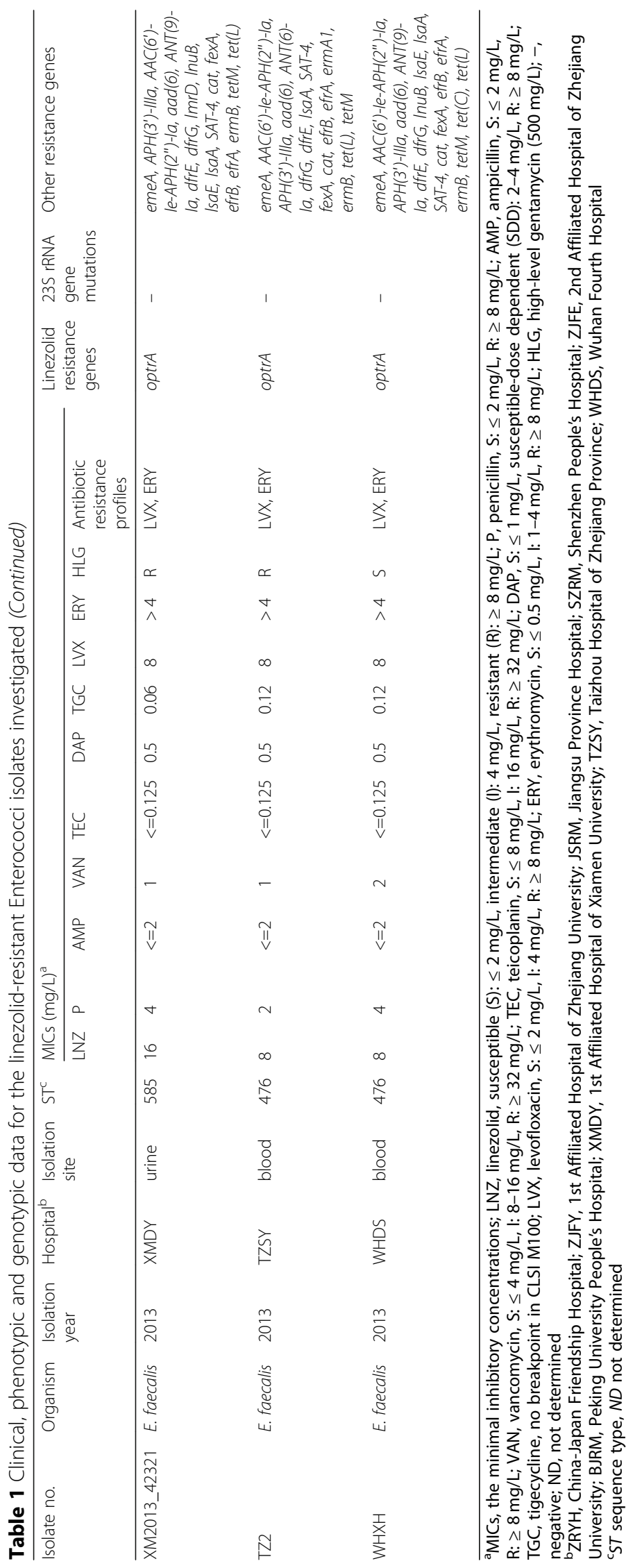


differences in the linezolid MICs between optrA-positive strains $(4-16 \mathrm{mg} / \mathrm{L})$ and optrA-negative strains $(8-16 \mathrm{mg} /$ L). Most of the optrA-positive strains also exhibited resistance to erythromycin $(16 / 16,100 \%)$, levofloxacin $(12 / 16$, $75 \%)$ and high-level gentamycin (500 mg/L) (13/16, 81.3\%). All strains were susceptible to vancomycin, teicoplanin, daptomycin and tigecycline. Three E.faecium and one E. faecalis strains $(4 / 16,25 \%)$ were resistant to penicillin and ampicillin, and all of 16 enterococci strains didn't possess beta-lactamase. Four strains (4/16, 25\%) belonged to MDR organism (Table 1).

\section{Distribution of antimicrobial resistance genes}

None of 16 linezolid-resistant enterococci strains contained cfr gene. Only one strain had the G2658 T mutation in 23S rRNA gene with linezolid MIC of $16 \mathrm{mg} / \mathrm{L}$. Most of the linezolid-resistant enterococci strains $(n=13)$ carried optrA gene (Table 1).

In addition to optrA genes, all optrA-positive strains harbored phenicols resistance gene fexA (13/13, 100\%), erythromycin resistance genes of different erm gene classes $($ ermA1, ermB) $(13 / 13,100 \%)$, trimethoprim resistant dihydrofolate reductase different $d f r$ gene classes $(d f r E$, $d f r G)(13 / 13,100 \%)$, ATP-binding cassette (ABC) antibiotic efflux pump different gene classes (lsaA, lsaE, efr $A$, efrB) (13/13, 100\%). Further, majority optrA-positive strains carried tetracycline resistance genes of different tet gene classes $(\operatorname{tet}[C]$, tet $[L]$, tet $M)(12 / 13,92.3 \%)$, multidrug and toxic compound extrusion (MATE) transporter emeA gene $(12 / 13,92.3 \%)$ and aminoglycosides inactivating enzyme different gene classes $\left(A A C\left(6^{\prime}\right)-I i\right.$, $A A C\left[6^{\prime}\right]-I e-A P H\left[2^{\prime \prime}\right]-I a, A P H\left[3^{\prime}\right]-I I I a$, aad [6], ANT[6]Ia, ANT[9]-Ia) (10/13, 76.9\%). Various additional resistance genes were identified including cat, $\ln u B, \ln u G$, $m d t F, S A T-4$ and efmA.

\section{Core-genome phylogenetic analysis}

The 12 E. faecalis isolates performed WGS were classified into 10 sequence types (STs): 3 ST476, 1 ST86, ST116, ST480, ST59, ST416, ST21, ST67, ST16 and ST585, respectively. One E. faecium isolate belonged to ST18.

The phylogenetic tree of $12 \mathrm{E}$. faecalis isolates harboring optrA gene showed that two of these isolates (29462 and XM2013_42321) were genetically unrelated with the rest isolates. Importantly, 1207_26W003 (Beijing), TZ2 (Zhejiang) and WHXH (Hubei) were recovered from different cities, were found very closely related (99.9\%), and all of 3 strains belonged to ST476. In addition, strain 19677 recovered from Guangdong was closely related (99.4\%) to strain 1202_13E004 recovered from Beijing. Further, strain 1203_10W003 isolated from Beijing and strain XM2013_71028 isolated from Fujian was closely related (99.3\%) (Fig. 1).

\section{Genetic environment of optrA on plasmids or chromosome}

Thirteen contigs containing the optrA gene were blasted in the GenBank database, and 10 contigs were mapped against the plasmids (pE121 [GenBank accession number KT862776] and pE419 [KT862777]). The size of these 10 contigs was between $6372 \mathrm{bp}$ and $21568 \mathrm{bp}$. According to the gene arrangements, the 10 contigs were divided into 4 groups: group 1 (29462 [MH225419], 1202 21W014 [MH225414]), group 2 (1203_10W003 [MH22 5415], SZ21494 [MH225420], ZJ11066 [MH225425]), group 3 (1207_26W003 [MH225416], 19677 [MH225418], XM2013_71028 [MH225424]), group 4 (WHXH [MH22 5422], XM2013_42321 [MH225423]). The genetic environment of optrA in Group 1 was similar to that of plasmid pE121 (KT862776). Compared to the plasmid pE121, ermA1 gene was absent and the rest of the sequences were almost identical. The genetic environment of optrA from Group 2 to Group 4 resembled that of plasmid pE419 (KT862777). Compared with pE419, the intergenic region between the left IS1216E and the first hypothetical protein was truncated in Group 2, two hypothetical proteins between optrA gene and the right IS1216E were missing in Group 3, and ermA1 gene and two hypothetical proteins were missing in Group 4. The common feature of genetic environment of optrA from Group 1 to Group 4 was flanked by IS1216E, and all of them carried phenicol resistance gene $f e x A$ and erythromycin resistance gene ermAl (Fig. 2a.).

The contigs containing optrA gene of 1202_13E004 (MH225413) (29141 bp), 19506 (MH225417) (22720 bp) and TZ2 (MH225421) (75117 bp) were mapped on chromosomal (CP008816). The strains 1202_13E004 and 19506 contained a transposon $\operatorname{Tn} 558$ (AJ715531) with three transposases and the resistance gene $f \operatorname{ex} A$, and the resistance gene optrA was adjacent to resistance gene ermA1. The strain TZ2 carried another transposon Tn554 (X03216) with three transposases and the resistance gene ermA1, and optrA was adjacent to resistance gene fexA (Fig. 2b.).

\section{Discussion}

This study indicates that the transferable resistance gene optrA is very prevalent among linezolid-resistant enterococci strains isolated from human. Much more optrA gene is located on plasmid than chromosome. The optrA gene located on plasmid is flanked by IS1216E, while that located on chromosome is mediated by transposons.

In this study, none of linezolid-resistant enterococci strains carried $c f r$, while most of them harbored optrA. This suggests that acquiring optrA is the main resistant mechanism in linezolid-resistant enterococci from human origin. The presence of optrA was limited to a 


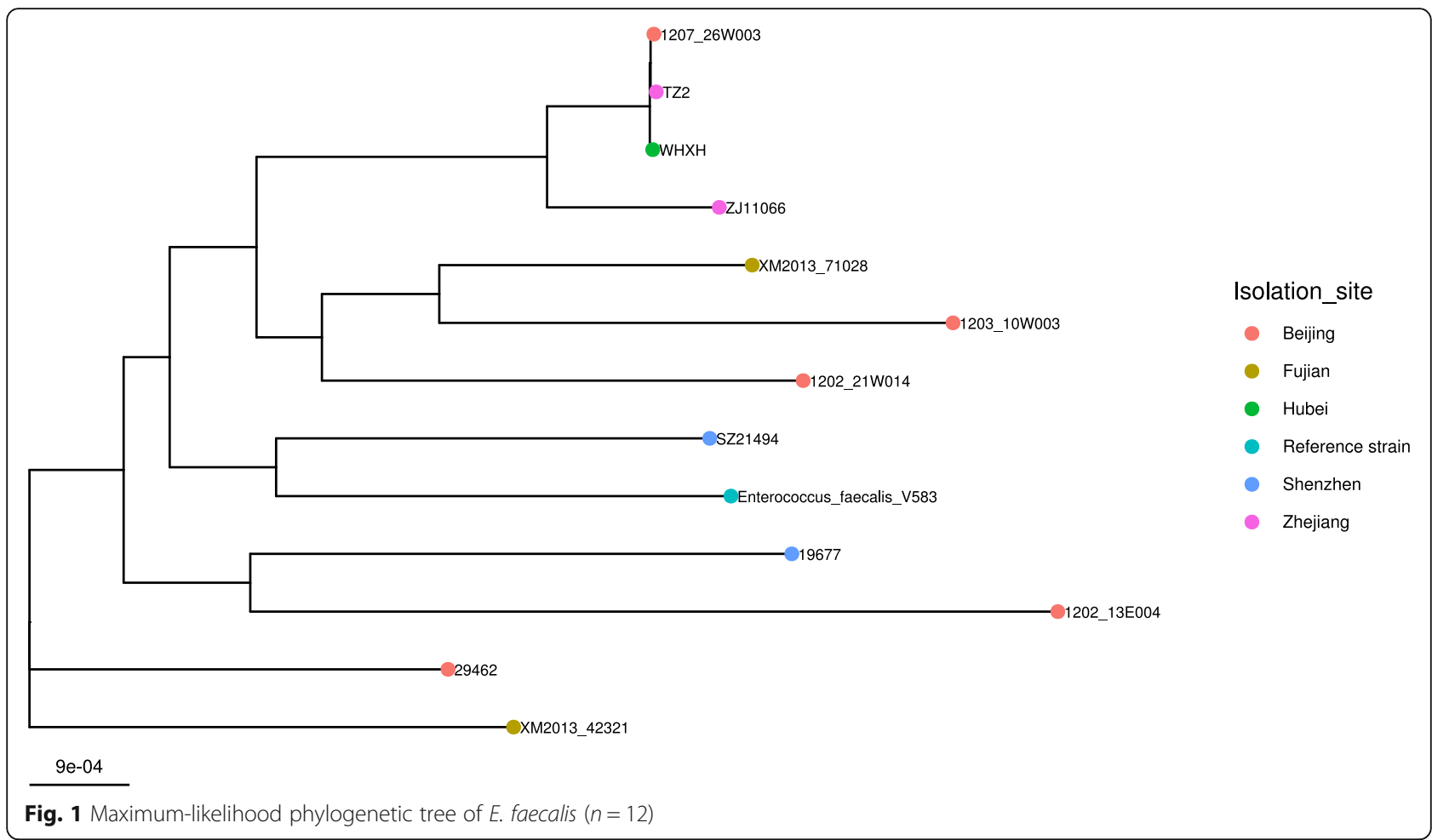

few species of the genus Enterococcus [35] and only rare species of Staphylococcus [4]. The surveillance studies indicated that only $3.9-6.2 \%$ of staphylococci strains were positive for optrA [4, 25], which suggests a low prevalence of this oxazolidinone resistance gene in the genus Staphylococcus.

In present study, the optrA gene was located on plasmids in most of enterococci strains. The optrA gene is often surrounded by insertion sequences when located on plasmids from enterococci strains. Our data showed that all of optrA found on plasmids were flanked by IS1216E, which was similar to a previous study [26]. Other studies also found that co-localization of optrA and cfr was close to IS21-558 and IS257 in S. sciuri [4, 27]. IS1216E belongs to the IS6 family which among other mediates transmission of the vancomycin resistance gene vanA in E. faecium, the oxazolidinone resistance gene $c f r$ in E. faecalis [36], the macrolide-lincosamide-streptogramin B resistance genes $\operatorname{erm}(B)$ and $\operatorname{erm}(T)$ in E. hirae [37] and Streptococcus gallolyticus subsp. pasteurianus [38], respectively, and the tetracycline resistance gene tet $(S)$ in Streptococcus infantis [39]. This indicates that optrA can be transferred between different genus bacteria by IS-mediated recombination events. Our study found that the optrA gene was located on chromosome in a few of enterococci strains. The optrA gene was adjacent to transposon $\operatorname{Tn} 558$ in two strains and to $\operatorname{Tn} 554$ in one strain. Tn558 was also detected upstream of optrA gene in $S$. sciuri and E. faecalis. The functionally active
Tn558 and Tn554 could excise from their host DNA and produce circular forms which precede the integration of the transposon into a new target sequence [40]. The similar genetic arrangement of Tn554 and optrA was identified in both of staphylococci and enterococci, which suggest optrA can be disseminated mediated by transposon between different genus bacteria. The optrA gene was flanked by insertion sequences or transposons, indicating that mobile genetic elements mediate horizontal transfer of optrA among different genus bacteria, which should be given more attention to avoid this novel oxazolidinone resistance gene dissemination in hospitals.

Our data showed the co-localization of resistance genes fexA $(n=13)$ and ermA1 $(n=9)$ with optrA. The gene fexA mediates resistance to fluorinated and non-fluorinated phenicols, which are widely used in livestock, but not in humans. The $f e x A$ gene was prevalent in florfenicol-resistant staphylcococci [4] and enterococci [23] from animal origin. The evidence of co-localization of fexA, ermA1 and optrA indicates that linezolid-resistant strains may be selected due to nonoxazolidinone antibiotics usage, such as macrolides (often used in hospital), florfenicol (often used in livestock) and et al.. The widespread use of florfenicol in livestock has exerted selective pressure on environmental bacteria and poses a significant public health threat to the increased resistance of the novel antibiotic linezolid.

In summary, optrA was found in most of linezolidresistant enterococci. The high diversity of optrA-carrying genetic platforms was found even in a limited 

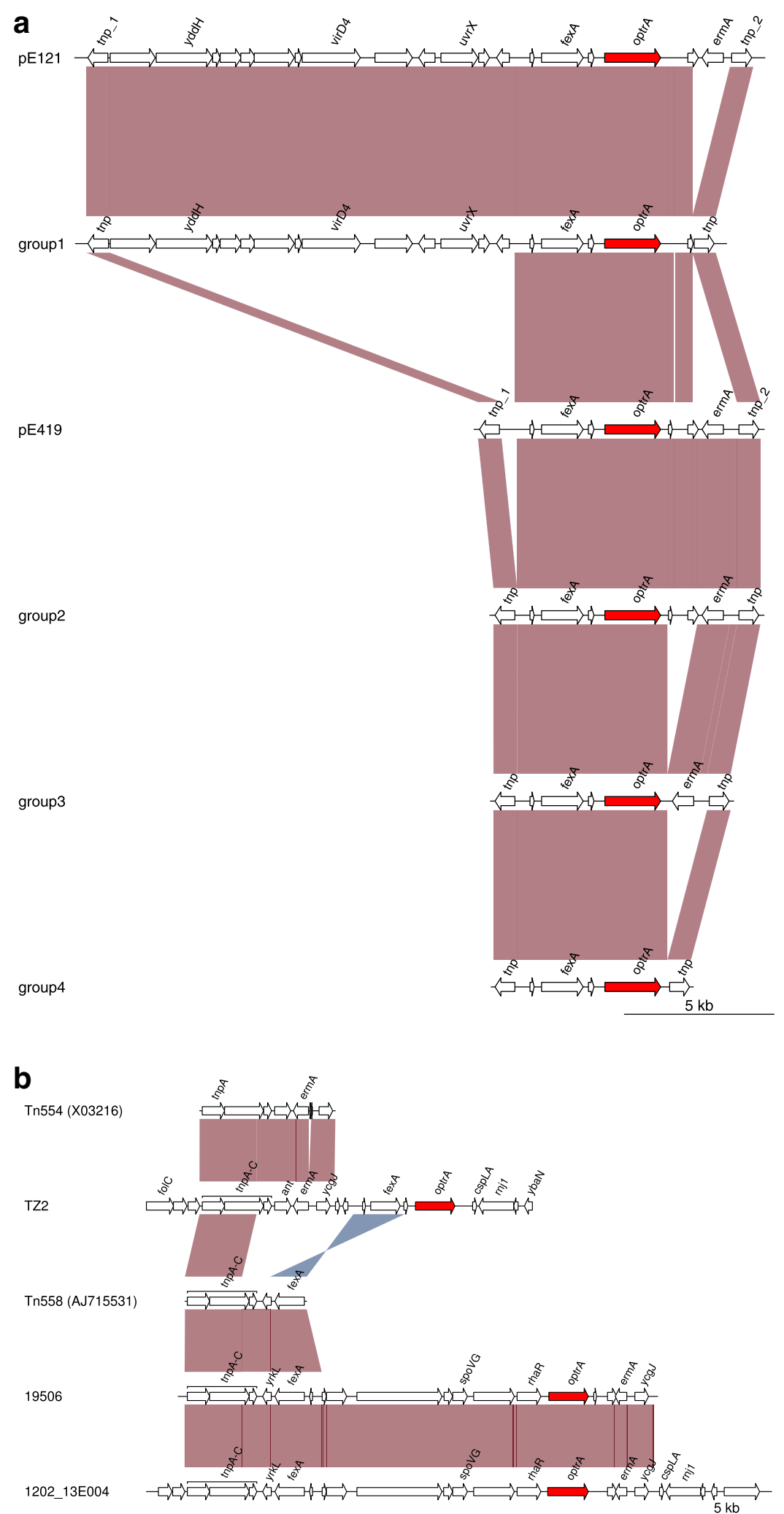

Fig. 2 (See legend on next page.) 
(See figure on previous page.)

Fig. 2 a Schematic presentation of the genetic environment of optrA-containing contigs mapped on plasmids in 10 enterococci isolates investigated in this study. $\mathbf{b}$ Schematic presentation of optrA-containing contigs mapped on chromosome in three enterococci isolates. Arrows indicate the positions and directions of transcription of the different genes. Genes with unknown functions are not marked. According to the gene arrangement, the 10 contigs mapped on plasmids were divided into 4 groups-group 1 (29462 [MH225419], 1202_21W014 [MH225414]), group 2 (1203_10W003 [MH225415], SZ21494 [MH225420], ZJ11066 [MH225425]), group 3 (1207_26W003 [MH225416], 19677 [MH225418], XM2013_71028 [MH225424]), group 4 (WHXH [MH225422], XM2013_42321 [MH225423])

number of analyzed isolates. The role of optrA in enterococci resistance to linezolid requires further investigation. The optrA gene was often flanked by insertion sequences or transposons, which might mediate the spread of optrA between different species or strains. The co-localization of $\operatorname{fexA}$, ermA1 and optrA suggests that linezolid-resistant enterococci can be selected by other antibiotics such as macrolides and so on, which should be given more attention in clinical practice.

\section{Conclusion}

We discovered the high diversity of optrA-carrying genetic platforms in our limited number of analyzed isolates. MGE mediated the dissemination of optrA between different species or strains. The optrA gene was found in most of the linezolid-resistant enterococci. Further studies should be done to clarify the linezolid resistance mechanism of optrA gene in Enterococcus species.

\section{Abbreviations}

ABC: ATP-binding cassette; cfr: chloramphenicol-florfenicol resistance; IS: Insertion sequences; MATE: Multidrug and toxic compound extrusion; MDR: Multi-drug resistant; MGE: Mobile genetic element; MICs: Minimal inhibitory concentrations; MRSA: Methicillin-resistant Staphylococcus aureus; PhLOPS A: Phenicols, lincosamide, oxazolidinones, pleuromutilin, and streptogramin A; VRE: Vancomycin-resistant enterococci; VRSA: Vancomycinresistant Staphylococcus aureus; WGS: Whole-genome sequencing

\section{Acknowledgments \\ The authors would like to thank Ji Zeng (Department of Clinical Laboratory, Wuhan Fourth Hospital), Qing Yang (Department of Clinical Laboratory, 1st Affiliated Hospital of Zhejiang University, Zhejiang University, Zhejiang, 310003, People's Republic of China), Rong Zhang (Department of Clinical Laboratory, 2nd Affiliated Hospital of Zhejiang University, Zhejiang University, Zhejiang, 310009, People's Republic of China), Xiaobo Ma (Department of Clinical Laboratory, 1st Affiliated Hospital of Xiamen University, Xiamen University, Fujian, 361003, People's Republic of China), Yingmei Liu (Department of Clinical Laboratory, China-Japan Friendship Hospital, Beijing, 100029, People's Republic of China), Weiyuan Wu (Department of Clinical Laboratory, Shenzhen People's Hospital, Shenzhen, 518020, People's Republic of China) and Dakang Hu (Department of Clinical Laboratory, Taizhou Hospital of Zhejiang Province, Zhejiang, 317000, People's Republic of China) for collecting the Linezolid- resistant strains.}

\section{Authors' contributions}

HW conceived and designed the study. HC, XW, YY, SL, YZ and QW performed experiments described in this study. $\mathrm{HC}$ wrote the draft, and $\mathrm{HW}$ revised it. All authors approved the final version.

\section{Funding}

This study was supported by the National Natural Science Foundation of China (No. 81471990).

\section{Availability of data and materials}

The sequences of the optrA-containing regions of 13 enterococci strains have been deposited at GenBank under the following accession numbers MH225413 (1202_13E004), MH225414 (1202_21W014), MH225415 (1203_10W003), MH225416 (1207_26W003), MH225417 (19506), MH225418 (19677), MH225419 (29462), MH225420 (SZ21494), MH225421 (TZ2), MH225422 (WHXH), MH225423 (XM2013_42321), MH225424 (XM2013_71028) and MH225425 (ZJ11066).

Ethics approval and consent to participate Not applicable.

\section{Consent for publication}

Not applicable.

\section{Competing interests}

The authors declare that they have no competing interests.

Received: 9 August 2018 Accepted: 30 June 2019

Published online: 12 July 2019

\section{References}

1. Brickner SJ, Barbachyn MR, Hutchinson DK, Manninen PR. Linezolid (ZYVOX), the first member of a completely new class of antibacterial agents for treatment of serious gram-positive infections. J Med Chem. 2008;51(7):1981-90. https:/doi.org/10.1021/jm800038g Epub 802008 Mar 800014

2. Meka VG, Pillai SK, Sakoulas G, Wennersten C, Venkataraman L, DeGirolami PC, Eliopoulos GM, Moellering RC Jr, Gold HS. Linezolid resistance in sequential Staphylococcus aureus isolates associated with a T2500A mutation in the $23 \mathrm{~S}$ rRNA gene and loss of a single copy of rRNA.J Infect Dis. 2004;190(2):311-7. https://doi.org/10.1086/421471 Epub 422004 Jun 421479.

3. Tsiodras S, Gold HS, Sakoulas G, Eliopoulos GM, Wennersten C, Venkataraman L, Moellering RC, Ferraro MJ. Linezolid resistance in a clinical isolate of Staphylococcus aureus. Lancet. 2001;358(9277):207-8. https://doi. org/10.1016/S0140-6736(01)05410-1.

4. Fan R, Li D, Fessler AT, Wu C, Schwarz S, Wang Y. Distribution of optrA and cfr in florfenicol-resistant Staphylococcus sciuri of pig origin. Vet Microbiol. 2017; 210:43-8. https://doi.org/10.1016/j.vetmic.2017.07.030 Epub 2017 Sep 1011.

5. RE M, LM D, RN J. Linezolid update: stable in vitro activity following more than a decade of. Drug Resist Updat. 2014;17(1-2):1-12.

6. $A M G, S S J, A R, L H ~ H, A ~ G, K L, B ~ V, F K$. Identification of 8-methyladenosine as the modification catalyzed by the radical. Rna. 2009;15(2):327-36.

7. Liu Y, Wang Y, Wu C, Shen Z, Schwarz S, Du XD, Dai L, Zhang W, Zhang Q, Shen J. First report of the multidrug resistance gene cfr in Enterococcus faecalis of animal origin. Antimicrob Agents Chemother. 2012;56(3):1650-4. https://doi.org/10.1128/AAC.06091-11 Epub 02011 Dec 06027.

8. Antonelli A, D'Andrea MM, Brenciani A, Galeotti CL, Morroni G, Pollini S, Varaldo PE, Rossolini GM. Characterization of poxtA, a novel phenicoloxazolidinone-tetracycline resistance. J Antimicrob Chemother. 2018;73(7): 1763-9.

9. Kehrenberg C, Schwarz S, Jacobsen L, Hansen LH, Vester B. A new mechanism for chloramphenicol, florfenicol and clindamycin resistance: methylation of $23 \mathrm{~S}$ ribosomal RNA at A2503. Mol Microbiol. 2005;57(4):1064-73. https://doi.org/10.1111/j.1365-2958.2005.04754.x.

10. Schwarz S, Werckenthin C, Kehrenberg C. Identification of a plasmid-borne chloramphenicol-florfenicol resistance gene in Staphylococcus sciuri. Antimicrob Agents Chemother. 2000;44(9):2530-3.

11. Dai L, Wu CM, Wang MG, Wang Y, Huang SY, Xia LN, Li BB, Shen JZ. First report of the multidrug resistance gene $\mathrm{cfr}$ and the phenicol resistance gene fexA in a Bacillus strain from swine feces. Antimicrob Agents Chemother. 2010;54(9): 3953-5. https://doi.org/10.1128/AAC.00169-10 Epub 02010 Jun 00128. 
12. Liu Y, Wang Y, Schwarz S, Li Y, Shen Z, Zhang Q, Wu C, Shen J. Transferable multiresistance plasmids carrying cfr in Enterococcus spp. from swine and farm environment. Antimicrob Agents Chemother. 2013;57(1):42-8. https:// doi.org/10.1128/AAC.01605-12 Epub 02012 Oct 01615.

13. Wang Y, Schwarz S, Shen Z, Zhou N, Lin J, Wu C, Shen J. Detection of the staphylococcal multiresistance gene $\mathrm{cfr}$ in macrococcus caseolyticus and Jeotgalicoccus pinnipedialis. J Antimicrob Chemother. 2012;67(8):1824-7. https://doi.org/10.1093/jac/dks163 Epub 2012 May 1810.

14. Wang Y, Li D, Song L, Liu Y, He T, Liu H, Wu C, Schwarz S, Shen J. First report of the multiresistance gene ffr in Streptococcus suis. Antimicrob Agents Chemother. 2013; 57(8):4061-3. https:/doi.org/10.1128/AAC.00713-13 Epub 02013 Jun 00713.

15. Wang Y, Wu CM, Schwarz S, Shen Z, Zhang W, Zhang Q, Shen JZ. Detection of the staphylococcal multiresistance gene $\mathrm{cfr}$ in Proteus vulgaris of food animal origin. J Antimicrob Chemother. 2011;66(11):2521-6. https://doi.org/1 0.1093/jac/dkr322 Epub $2011 \mathrm{Jul} 2526$.

16. Wang Y, He T, Schwarz S, Zhou D, Shen Z, Wu C, Ma L, Zhang Q, Shen J. Detection of the staphylococcal multiresistance gene $\mathrm{cfr}$ in Escherichia coli of domestic-animal origin. J Antimicrob Chemother. 2012;67(5):1094-8. https://doi.org/10.1093/jac/dks020 Epub 2012 Feb 1013.

17. Chen H, Yang Q, Zhang R, He W, Ma X, Zhang J, Xia F, Zhao F, Cao J, Liu Y, et al. In vitro antimicrobial activity of the novel oxazolidinone tedizolid and comparator agents against Staphylococcus aureus and linezolid-resistant gram-positive pathogens: a multicentre study in China. Int J Antimicrob Agents. 2014;44(3):276-7. https://doi.org/10.1016/j.jjantimicag.2014.05.007 Epub 2014 Jun 1023.

18. Shen J, Wang Y, Schwarz S. Presence and dissemination of the multiresistance gene $\mathrm{cfr}$ in gram-positive and gram-negative bacteria. J Antimicrob Chemother. 2013;68(8):1697-706. https://doi.org/10.1093/jac/ dkt092 Epub 2013 Mar 1629.

19. Deshpande LM, Ashcraft DS, Kahn HP, Pankey G, Jones RN, Farrell DJ, Mendes RE. Detection of a new cfr-like gene, $c f r(B)$, in Enterococcus faecium isolates recovered from human specimens in the United States as part of the SENTRY antimicrobial surveillance program. Antimicrob Agents Chemother. 2015;59(10):6256-61. https://doi.org/10.1128/AAC.01473-15 Epub 02015 Jul 01427.

20. Hansen $L H$, Vester B. A cfr-like gene from Clostridium difficile confers multiple antibiotic resistance by the same mechanism as the $\mathrm{cfr}$ gene. Antimicrob Agents Chemother. 2015;59(9):5841-3. https://doi.org/10.1128/ AAC.01274-15 Epub 02015 Jul 01276

21. Tang Y, Dai L, Sahin O, Wu Z, Liu M, Zhang Q. Emergence of a plasmidborne multidrug resistance gene $\mathrm{cfr}(\mathrm{C})$ in foodborne pathogen campylobacter. J Antimicrob Chemother. 2017;72(6):1581-8. https://doi. org/10.1093/jac/dkx023.

22. Shore AC, Brennan OM, Ehricht R, Monecke S, Schwarz S, Slickers P, Coleman DC. Identification and characterization of the multidrug resistance gene cfr in a Panton-valentine leukocidin-positive sequence type 8 methicillin-resistant Staphylococcus aureus IVa (USA300) isolate. Antimicrob Agents Chemother. 2010;54(12):4978-84. https://doi.org/10.1128/AAC. 01113-10 Epub 02010 Oct 01114

23. Wang Y, Lv Y, Cai J, Schwarz S, Cui L, Hu Z, Zhang R, Li J, Zhao Q, He T, et al. A novel gene, optrA, that confers transferable resistance to oxazolidinones and phenicols and its presence in Enterococcus faecalis and Enterococcus faecium of human and animal origin. J Antimicrob Chemother. 2015;70(8):2182-90. https://doi.org/10.1093/jac/dkv116 Epub 2015 May 2114.

24. Cai J, Wang Y, Schwarz S, Lv H, Li Y, Liao K, Yu S, Zhao K, Gu D, Wang $X$, et al. Enterococcal isolates carrying the novel oxazolidinone resistance gene optrA from hospitals in Zhejiang, Guangdong, and Henan, China, 2010-2014. Clin Microbiol Infect. 2015;21(12):1095.e1091-4. https://doi.org/10.1016/j.cmi.2015.08.007 Epub 2015 Aug 1028.

25. Fan R, Li D, Wang Y, He T, Fessler AT, Schwarz S, Wu C. Presence of the optrA gene in methicillin-resistant Staphylococcus sciuri of porcine origin. Antimicrob Agents Chemother. 2016;60(12):7200-5. https://doi.org/10.1128/ AAC.01591-16 Print 02016 Dec.

26. He T, Shen Y, Schwarz S, Cai J, Lv Y, Li J, Fessler AT, Zhang R, Wu C, Shen J, et al. Genetic environment of the transferable oxazolidinone/phenicol resistance gene optrA in Enterococcus faecalis isolates of human and animal origin. J Antimicrob Chemother. 2016;71(6):1466-73. https://doi.org/1 0.1093/jac/dkw016 Epub 2016 Feb 1421
27. Li D, Wang Y, Schwarz S, Cai J, Fan R, Li J, Fessler AT, Zhang R, Wu C, Shen $\mathrm{J}$. Co-location of the oxazolidinone resistance genes optrA and $\mathrm{cr}$ on a multiresistance plasmid from Staphylococcus sciuri. J Antimicrob Chemother. 2016;71(6):1474-8. https://doi.org/10.1093/jac/dkw040 Epub 2016 Mar 1476

28. Chen H, Wu W, Ni M, Liu Y, Zhang J, Xia F, He W, Wang Q, Wang Z, Cao B, et al. Linezolid-resistant clinical isolates of enterococci and Staphylococcus cohnii from a multicentre study in China: molecular epidemiology and resistance mechanisms. Int J Antimicrob Agents. 2013;42(4):317-21.

29. Mendes RE, Deshpande LM, Farrell DJ, Spanu T, Fadda G, Jones RN. Assessment of linezolid resistance mechanisms among Staphylococcus epidermidis causing bacteraemia in Rome, Italy. J Antimicrob Chemother. 2010;65(11):2329-35. https://doi.org/10.1093/jac/dkq331 Epub 2010 Sep 2314.

30. Nurk S, Bankevich A, Antipov D, Gurevich AA, Korobeynikov A, Lapidus A, Prjibelski AD, Pyshkin A, Sirotkin A, Sirotkin $Y$, et al. Assembling single-cell genomes and mini-metagenomes from chimeric MDA products. J Comput Biol. 2013;20(10):714-37. https://doi.org/10.1089/cmb.2013.0084.

31. Seemann T. Prokka: rapid prokaryotic genome annotation. Bioinformatics. 2014;30(14):2068-9. https://doi.org/10.1093/bioinformatics/btu2153 Epub 2014 Mar 2018

32. Siguier P, Perochon J, Lestrade L, Mahillon J, Chandler M. ISfinder: the reference centre for bacterial insertion sequences. Nucleic Acids Res. 2006; 34(Database issue):D32-6. https://doi.org/10.1093/nar/gkj014.

33. Zankari E, Hasman $H$, Cosentino S, Vestergaard M, Rasmussen S, Lund O, Aarestrup FM, Larsen MV. Identification of acquired antimicrobial resistance genes. J Antimicrob Chemother. 2012;67(11):2640-4.

34. Stamatakis A. RAxML-VI-HPC: maximum likelihood-based phylogenetic analyses with thousands of taxa and mixed models. Bioinformatics. 2006;22(21):2688-90. https://doi.org/10.1093/ bioinformatics/btl446 Epub 2006 Aug 2623.

35. Cui L, Wang Y, Lv Y, Wang S, Song Y, Li Y, Liu J, Xue F, Yang W, Zhang J. Nationwide surveillance of novel Oxazolidinone resistance gene optrA in Enterococcus isolates in China from 2004 to 2014. Antimicrob Agents Chemother. 2016;60(12):7490-3. https://doi.org/10.1128/AAC.01256-16 Print 02016 Dec.

36. Darini AL, Palepou MF, Woodford N. Effects of the movement of insertion sequences on the structure of VanA glycopeptide resistance elements in Enterococcus faecium. Antimicrob Agents Chemother. 2000;44(5):1362-4.

37. Raze D, Dardenne O, Hallut S, Martinez-Bueno M, Coyette J, Ghuysen JM. The gene encoding the low-affinity penicillin-binding protein $3 r$ in Enterococcus hirae S185R is borne on a plasmid carrying other antibiotic resistance determinants. Antimicrob Agents Chemother. 1998;42(3):534-9.

38. Tsai JC, Hsueh PR, Chen HJ, Tseng SP, Chen PY, Teng LJ. The erm(T) gene is flanked by IS1216V in inducible erythromycin-resistant Streptococcus gallolyticus subsp. pasteurianus. Antimicrob Agents Chemother. 2005;49(10): 4347-50. https://doi.org/10.1128/AAC.49.10.4347-4350.2005

39. Ciric L, Brouwer MS, Mullany P, Roberts AP. Minocycline resistance in an oral Streptococcus infantis isolate is encoded by tet(S) on a novel small, low copy number plasmid. FEMS Microbiol Lett. 2014;353(2):106-15. https://doi. org/10.1111/1574-6968.12410 Epub 12014 Mar 12419.

40. Kehrenberg C, Schwarz S. Florfenicol-chloramphenicol exporter gene fexA is part of the novel transposon Tn558. Antimicrob Agents Chemother. 2005; 49(2):813-5. https://doi.org/10.1128/AAC.49.2.813-815.2005.

\section{Publisher's Note}

Springer Nature remains neutral with regard to jurisdictional claims in published maps and institutional affiliations. 